\title{
LA INCIDENCIA LOCAL DE LAS ORGANIZACIONES DE LA ECONOMÍA SOCIAL Y SOLIDARIA: APORTES DESDE UNA PERSPECTIVA RELACIONAL
}

Revista Trama

Volumen 7, número 2

Julio - Diciembre 2018

Páginas 71-85

ISNN-1659-343X

http://revistas.tec.ac.cr/trama
A Incidência local das organizações da economia social e solidária: contribuições de uma perspectiva relacional

Óscar Segura Castro ${ }^{1}$

Fecha de recepción: 16 de agosto de 2018 Fecha de aprobación: 12 de setiembre de 2018

Segura, O. (2018). La incidencia local de las organizaciones de la economía social y solidaria: aportes desde una perspectiva relacional, Trama, revista de ciencias sociales y humanidades, Volumen 7, (2), págs. 71-85.

DOI: https://doi.org/10.18845/tramarcsh.v7i2.3946
1. Investigador en el Centro Dominico de Investigación (Heredia, Costa Rica). Licenciado en Dirección de Empresas (UCR), posee un curso de posgrado en Economía Social y Solidaria y Desarrollo Local (Universidad Nacional de General Sarmiento, Buenos Aires Argentina) y es egresado de la Maestría en Ciencias Políticas (UCR). 


\section{Resumen}

El presente artículo ofrece una ventana para comprender más ampliamente el fenómeno de las organizaciones de la Economía Social y Solidaria (ESS), particularmente cómo estas se relacionan con sus entornos más próximos. El punto de partida se ofrece desde una perspectiva teórica que aporta a una comprensión sistémica del fenómeno empresarial. Lo anterior logra articularse con las particularidades del fenómeno empresarial propiamente asociativo, que por sus cualidades se conecta de un modo particular en el espacio local. Así, resulta relevante precisar que las organizaciones de la ESS, por su habitual arraigo no se instalan, sino que surgen desde el territorio. La actividad productiva no se realiza en el vacío, sino que la realizan personas concretas vinculadas en un determinado espacio. Esto deja observar que los efectos de la actividad productiva no son accidentales, sino que están marcados por condiciones previas que son estructurales. Cómo afecta una organización su entorno, tendría que pasar primero por preguntarse cómo participan las personas que habitan ese entorno de esa organización, o sea, del tipo que esta sea. De esto se sigue observar las formas en las que dicha relación se materializa, pues ello permite comprender y dar cuenta del efecto de la actividad productiva, pero también hace posible comprenderlo más allá de lo habitualmente entendido como "económico". Esto, que es una suerte de aporte propiamente metodológico, no sería posible sin una particular lectura teórica y epistemológica del fenómeno empresarial en general y del empresarial asociativo en particular.

Palabras clave: cooperativas, economía social y solidaria, desarrollo local, stakeholders.

\section{Resumo}

O presente artigo busca compreender de forma mais amplia o fenômeno das organizações da Economia Social e Solidária (ESS) e particularmente busca entender como estas se relacionam com o seu entorno mais próximo. O ponto de partida nos oferece uma perspectiva teórica que guia a uma compreensão sistemática do fenômeno empresarial. Tal perspectiva alcança uma articulação entre as particularidades do fenômeno empresarial propriamente associativo e o espaço local, essa conexão ocorre de forma particular e por meio das qualidades especificas do já mencionado fenômeno. Assim, resulta relevante, especificar que as organizações da ESS por sua habitual origem não se instalam senão que surgem desde o território. A atividade produtiva não se realiza no vazio nem a realizam pessoas anônimas ou desvinculadas desse espaço. Isto nos permite observar que os efeitos da atividade produtiva não são acidentais e sim que estão marcados pelas condições prévias que são estruturantes. Como afeta uma organização o seu entorno? Antes dessa pergunta vale a pena questionar primeiramente, como participam as pessoas que habitam o entorno dessa organização. A partir desses questionamentos é possível observar as formas nas quais dita relação se materializam, pois isso permite compreender o fenômeno estudado para além do que habitualmente é entendido como "econômico". Este trabalho traz uma clara colaboração propriamente metodológica, porém que não seria possível sem uma particular leitura teórica e epistemológica do fenômeno empresarial em geral e do empresarial associativo em particular.

Palavras-chave: cooperativas, economia social e solidária, desenvolvimento local, stakeholders. 


\section{INTRODUCCIÓN}

Este trabajo es una suerte de análisis ampliado que se deriva del conjunto de hallazgos de un trabajo de investigación anterior, titulada "Cooperativas y su impacto al desarrollo. Reconociendo vías y formas de incidencia" (Céspedes y Segura, 2017) ${ }^{2}$. En cierto modo se retoma el marco teórico de dicha investigación, pero con algunas precisiones en función de explicitar cómo la dinámica relacional propia de las organizaciones cooperativas y de la Economía Social y Solidaria (ESS), a partir de sus cualidades asociativo-democráticas, crean una realidad altamente compleja de relaciones $y$ efectos comunitarios que es posible identificar con mayor claridad en el espacio local. Esta relación requiere ser comprendida y explicada adecuadamente para develar las posibilidades creadas a partir de estos hallazgos antes mencionados; pero, más allá de ellos individualmente, se buscar asomar a cómo las vías y formas de impacto sugieren un modo de ser y de relacionarse para crear realidades sociales, políticas, culturas y económicas relevantes, las cuales requieren ser reconocidas y comprendidas.

Para lograr lo anterior hay que considerar que la dimensión social de lo que primeramente se presenta como un fenómeno económico, puede resultar como una tarea quizás escurridiza o con alguna dificultad incluso epistemológica. Cómo se entiende el fenómeno, sus partes y la relación entre ellas, eso puede ser una primera dificultad; seguidamente, vendrían obstáculos metodológicos sobre cómo abordar y hacer inteligible esto que se desea conocer. Sin duda son retos interesantes y que ofrecen posibilidades para dar cuenta de dimensiones opacas de los fenómenos sociales en general, pero en particular de aquellos usualmente apropiados por el pensamiento económico dominante.

De esta manera se ubica a las organizaciones cooperativas, y de la Economía Social y Solidaria (ESS), dentro de esta concepción amplia y rica de relaciones sociales. Para comprenderlas y abordarlas, el presente trabajo intenta hacerlas visibles dentro del espacio como escenario propicio para las relaciones sociales, económicas, políticas y culturas de las que estas organizaciones forman parte. A pesar de esto, se vuelve necesario, entonces, conocer de estas relaciones, el con quiénes, el para qué y qué efectos resultan. Pero, antes que solo fijarse en los efectos o en detectarlos, en este trabajo en particular lo que se busca, es ofrecer una comprensión de las condiciones particulares que hay detrás de ellos. Así, acercamos analíticamente "comunidad" y "organización" como dos elementos, que como se verá, se les debe entender en su profunda y compleja co-existencia y relación en contextos espacialmente dados.

\section{LA ASOCIATIVIDAD COMO PARTICULARIZADOR DEL FENÓMENO PRODUCTIVO}

El fenómeno cooperativo $y$, en general, el mundo de las organizaciones productivas de carácter asociativo-democrático de la ESS, posee cualidades que podrían considerarse como particulares. Por una parte, sus cualidades internas de organización y funcionamiento de carácter asociativo-democrático serían un primer elemento distintivo. En este sentido, se trataría de un fenómeno propiamente empresarial determinado a partir de sus cualidades internas. Pero, más allá de sí mismas, cualquier unidad económica, del tipo que sea, posee una condición más amplia que viene de su naturaleza social. Se trata de un fenómeno económico inscrito socialmente, por lo tanto, ubicable espacialmente y reconocible como generador de relaciones en ese espacio.

Lo anterior es una constatación que, incluso, podría resultar muy obvia. Además, también cierta para el conjunto de las empresas, incluso para aquellas que operan en contextos globales, pues no pueden eximirse de estas cualidades que las conectan a un -o a muchos- espacio y a otros agentes junto a quienes cohabitan inevitablemente. Ahora bien, esta existencia de carácter espacial, siendo que es un rasgo inevitable, tiene también expresiones concretas para las diferentes formas de organización empresarial. Estos rasgos concretos vienen dados por la compleja relación entre las organizaciones empresariales y el espacio desde el cual existen y se desarrollan. Hablamos de una relación viva y compleja, altamente dinámica y determinada en buena parte por aspectos extra-económicos no siempre reconocibles. No se trata de "medir efectos" solamente, sino de reconocer la complejidad detrás, de observar y comprender la composición de las organizaciones como parte de sistemas espaciales y de relaciones que se realizan simultáneamente y que se construyen y afectan mutuamente.

Dado lo anterior, el presente artículo parte de reconocer este mundo complejo en el que existen y se relacionan las empresas cooperativas y las organizaciones de la Economía Solidaria en general. Lo cual, como se verá a lo largo de este trabajo, no es una premisa sustentada sin base empírica. El carácter asociativo-democrático de estas organizaciones es una expresión de otras relaciones sociales, que las anteceden y las suceden, pero que en definitiva les

2. Este artículo es producto tanto de un trabajo de investigación en el que participó el autor en el marco del convenio entre el Centro Dominico de investigación y el Instituto Nacional de Fomento Cooperativo, como de un proceso de análisis posterior realizado por el autor como parte del proyecto "Efecto de las organizaciones de economía solidaria y comercio justo sobre el desarrollo económico y social de las comunidades en Costa Rica" de la Escuela de Economía de la Universidad Nacional, Heredia, Costa Rica. 
provee de una impronta peculiar, distinta de las que se pueden encontrar en otras formas de organización empresarial. Es por esto por lo que resulta esclarecedor ese mundo de relaciones, tanto en sus formas como en aquello que compone su carácter particular. De esta manera, procuraremos recorrer los aspectos teóricos y metodológicos que, desde nuestra perspectiva, hacen posible comprender el fenómeno de la Economía Social y Solidaria como uno particular y con importantes potencialidades para el desarrollo del $-y$ desde- el espacio local.

\section{LA PRÁCTICA EMPRESARIAL Y SUS CONTRADICCIONES}

El fenómeno empresarial como actividad humana que se suscribe en contextos concretos, sean locales y/o globales, no es posible abstraerlo ni de relaciones entre actores sociales ni de una ubicación espacial determinada. No hay empresa, sea del tipo que sea, que opere con independencia de actores a los que su actividad afecta y de un medio ambiente que también recibe el impacto de su actividad. Lo anterior podría ser tomado como una constatación que no admite discusión, lo cierto es que la posición de Milton Friedman, aunque podría haberse convertido en "políticamente incorrecta", no es menos susceptible de estar incluso normalizada dentro de las lógicas operacionales de empresas en la actualidad. Para este autor, las empresas no tienen mayor responsabilidad social que la de generar valor para sus accionistas; a su vez, menciona algunas pautas mínimas legales y de "competencia leal" que se deben respetar (Friedman, 1966, p. 173), nada más. En general se trata de una postura auto-referenciada de la empresa, como si esta existiera en una suerte de vacío, que solo podría ser ocupado por sus propios intereses, pues nada interesa fuera de ella misma y de su propósito último.

También, Adela Cortina, señala la pretendida condición "amoral" que algunas corrientes de pensamiento han querido imprimir a la actividad empresarial. Para ello se refiere a tres líneas de influencia ideológicas principalmente. De ellas, se quisiera hacer referencia solamente a dos, dada la pertinencia concreta de estas con la línea argumentativa en este punto. En primera instancia se refiere al positivismo 3 , que deriva del postulado de que la economía no puede mezclarse con valoraciones subjetivas y que debe pretender siempre objetividad ${ }^{4}$; la segunda, que hace referencia a las teorías de las organizaciones, que sugieren que las personas, y no las organizaciones, son objeto de una exigencia moral (1997, pp. 19-23). Ambas corrientes, en el contexto de la práctica económica de las empresas, son las que pueden tener mayor vigencia,

3. Sobre esta discusión hay varios trabajos de Jorge Arturo Chaves, en particular su libro “De la Utopía a las políticas económicas. Para una ética de las políticas económicas" (1999, pp. 183-194), en donde se desarrolla con amplitud este aspecto de lo que él mismo considera "una antigua y amplia discusión". Se refiere, propiamente, a lo 'normativo' y a lo 'positivo' en economía. Para él, hay un "sinsentido y un peligro" en pretender que existe "una economía pura, libre de contaminaciones políticas, ideológicas y éticas." (p. 186). Es útil el desarrollo que al respecto hace Chaves, pues permite contextualizar la discusión al mostrar que "el tema de la distinción entre economía positiva y economía normativa [...] puede encontrarse como objeto de discusión desde los tiempos de Senior o J. S. Mill, hasta los recientes de Samuelson o Myrdal, pasando por Walras, Pareto y otros autores no menos importantes." (p. 184). Finalmente, lo que este autor busca es cimentar su argumento en los aportes especialmente de Myrdal, precisamente porque su interés es mostrar la necesaria consideración de lo ético al interior, como componente intrínseco, del proceso mismo de las decisiones económicas. Justamente es por ahí es donde transita Myrdall, al intentar señalar los peligros de pretender una ciencia económica desconectada de la política y en general de los valores. No sobra decir que décadas después, otro Premio Nobel, Amartya Sen, trata ampliamente sobre la conexión entre ética y economía (Sobre ética y economía, 1989, Alianza Editorial), que como hemos visto en su raíz tiene esta discusión entre lo positivo y lo normativo en economía.

4. Un referente operativo, por decirlo de alguna manera, sobre esta corriente a la empresa, o más precisamente, a la idea de cómo se entiende la empresa como parte de la sociedad, la ofrece el mismo Friedman (ver cita anterior, Friedman, 1966). Este es un ejemplo de cómo la concepción positivista de la economía como ciencia se traslada a la actividad económica, concretamente a la actividad de un agente económico. Así las cosas, desde esta posición, la empresa no es objeto de una condición moral que pueda o deba resignificar de alguna manera su posición y su rol en la sociedad. Vemos así, la concreción operativa de una idea que, en nuestro criterio, condiciona la posibilidad de que epistemológicamente se pueda concebir de un modo diferente la empresa como objeto mismo de estudio. 
aún a pesar de algunas corrientes que contravienen, de una u otra manera, esta supuesta condición aséptica de las empresas, en cuanto a alguna exigencia de comportamiento moral o responsabilidad frente a la sociedad. Un buen ejemplo de estas corrientes, a pesar de las limitaciones que puedan reconocérsele, es la llamada Responsabilidad Social Empresarial (RSE).

La cuestión primordial, en este punto, es reconocer que la práctica no está libre de controversia respecto a la idea de cómo las empresas responden frente a sus responsabilidades con la sociedad, o si de hecho las tienen. Hay ejemplos dramáticos, como los ocurridos en Asia, especialmente asociados a la industria textil, donde incendios muy relacionados a las pésimas condiciones laborales han ocasionado cientos de muertes. El más grande de los últimos años acontecido en Bangladesh en 2013 (El Mundo, 2013), con un total de 300 personas muertas. Pero, en Costa Rica, la situación laboral no es favorable para el $60 \%$ de las personas asalariadas, como señalaba el Programa Informe Estado de la Nación en su decimonoveno informe (2013); esta porción de personas trabajadoras veía incumplido alguno de los derechos laborales garantizados por ley. Todo esto para indicar que, a pesar de ciertas corrientes de pensamientos que señalan la responsabilidad de la actividad empresarial frente a la sociedad y al medio ambiente, hechos lamentables siguen sucediéndose. La práctica es elocuente al expresar cómo aquella postura -énfasis en la rentabilidad de los accionistas- que en la década de los sesenta defendía Friedman, sigue vigente, tal vez no explícitamente por su grotesco peso discursivo, pero sí en la práctica.

No se busca afirmar que toda la práctica empresarial no integra otros intereses que los propios, sino solamente que ante lo que podría pensarse que es una idea de consenso, no en pocos contextos cuando se pone el foco en la práctica, esta no integra plenamente las consecuencias de su actividad y el efecto negativo que esta puede tener en poblaciones directamente afectadas por ella. Desde luego, también hay que hacer referencia al ambiente como una de esas "poblaciones". En el tanto se pase por alto el destrozo de derechos laborales y del medio ambiente, nos parece que no hay claridad acerca de que la práctica empresarial, necesariamente, va más a allá de la generación de valor para los accionistas. Aunque, como se dijo antes, el peso discursivo de reconocer algo como esto es muy fuerte, la práctica continúa mostrando la existencia de incongruencias que sugieren un consenso que continúa sin alcanzarse en todos sus extremos. La práctica empresarial, por los intereses que convergen en ella, es un terreno en disputa y alberga comportamientos que cuando menos no son claros respecto al modo en cómo se integran los intereses externos que esta afecta.

\section{DE LA TEORÍA DE LOS STAKEHOLDERS A UN ENFOQUE RELACIONAL}

Fundamentar que es posible aproximarse al conocimiento de los efectos de las organizaciones de la ESS con las comunidades, requiere explicitar por qué vías y bajo qué formas se materializan estos efectos. El primer paso sería señalar a través de qué medios los efectos se trasladan hasta sus destinatarios. Diremos que, en un enfoque relacional, serán, naturalmente, las relaciones. Por ello será necesario develar con quiénes y en cuáles condiciones se realizan estas. Pero, para lograr esto, conviene referirse a algunas cualidades esenciales que son propias de la actividad empresarial en general, para luego marcar el terreno específico al que refiere nuestro objeto particular dentro de ese universo más amplio de empresas. Como un punto de partida general, la teoría de los stakeholders Ilama la atención sobre aspectos que son altamente relevantes para comprender esta dinámica relacional que se ha venido mencionando. Esta teoría a pesar de que es una vía de acceso metodológico para propuestas como la de Responsabilidad Social Empresarial (RSE), no es en esta línea en la que, pensamos, se inscribe el propósito de este artículo. En todo caso, la teoría de los stakeholders tiene la utilidad de proveer elementos de partida que son esenciales para comprender la actividad empresarial como una actividad profundamente social. Creemos que no hay otro modo de entenderla, pero, como se ha señalado antes, no es así siempre y, sobre todo, no es así siempre en la práctica.

Elsa González ofrece una interesante aproximación. Para ella esta teoría es un puente "para que la ética empresarial dialógica pueda pasar de la teoría a la práctica" (2007, p. 208). En el marco de este artículo, se atienden a otros propósitos y se utilizará, especialmente, la definición que González toma de Richard Edward Freeman, para poner el foco sobre lo que es un aspecto fundamental, la definición misma de stakeholder. A saber, un stakeholder es "cualquier grupo o individuo que puede afectar o ser afectado por el logro de los objetivos de la empresa" (Freeman, citado por González, 2007, p. 208). 
En primera instancia, en la definición anterior, hay un elemento implícito que resulta de gran interés: la empresa, como organización social que realiza una actividad interconectada con sujetos (personas, organizaciones, etc.) concretos. Aunque parece obvio, sugiere la existencia de otros actores que son relevantes más allá de la empresa misma, en el tanto son afectados por la actividad de esta. Incluso, para Adela Cortina, todos los afectados -directos o indirectospor la actividad de una empresa son interlocutores válidos con intereses que merecen tenerse en cuenta en la toma de decisiones (1997, p. 29). Por lo que, no solo se trata de reconocer que existen estos "afectados potenciales", sino que poseen intereses legítimos en el tanto son afectados por la actividad de la empresa. Ahora bien, continuando con González, hay tres razones principales por las que esta teoría es de interés: 1) porque permite pensar un nuevo paradigma de la empresa, crea la comprensión de "la empresa plural", más allá de accionistas y trabajadores; 2) porque entre los distintos stakeholders que conforman la empresa se establecen relaciones o, como dice González, "entre los distintos stakeholders que configuran la organización empresarial existen expectativas recíprocas de comportamiento"; y 3) finalmente, porque permite divisar la responsabilidad social de la empresa (González, 2007, p. 208-209).

Los puntos 1 y 2 son aspectos especialmente iluminadores en el marco de la idea que se viene procurando sustentar. Lograr reconocer que la empresa es relevante más allá de los accionistas y de quienes laboran en ella es un primer paso en absoluto necesario. Abre a una concepción en la que se visibilizan otros actores para quienes legítimamente la actuación de la empresa no les es indiferente; así, queda abierta la posibilidad de identificar relaciones entre esos stakeholders, que es a lo que hace referencia el punto 2. De esta manera es posible comprender no solo a la empresa más allá de sí misma sino las relaciones que entre ella y esos actores y/o personas se tejen y se entretejen. Se trata de hacer evidente la profunda naturaleza social de la empresa. A partir de ella es posible pensar en aproximaciones con metodologías apropiadas. Entendemos que estas relaciones se materializan mediante esa dinámica relacional a través de las múltiples formas y vías concretas que deben ser identificadas. Para ello es fundamental antes identificar con quiénes se relaciona y para qué.

\section{LAS EMPRESAS (Y TAMBIÉN LAS ORGANIZACIONES DE LA ESS) COMO SISTEMAS ABIERTOS}

Es cierto que la actividad de la empresa, cualquiera que sea, por su naturaleza misma, tiene diversidad de efectos en el espacio donde se instala o desde el cual surge. El carácter social de ésta, como se ha visto, lo confirma. Este refiere a una condición que es propia de la empresa como un actor social relevante, al respecto la observación que hace Elsa González permite dar un paso más en la tarea de dimensionar de mejor manera a la empresa, así como su modo de estar presente y de interactuar en el espacio social:

la concepción de la empresa, como una institución social inscrita en un sistema abierto, dinámico y de interdependencias entre sus instituciones, muestra que ésta actúa dentro de sistemas sociales, político-administrativos y económicos que imponen límites o constricciones al actuar de la empresa pero que también se ven modificados por las políticas, estrategias y valores de la empresa. (2007, p. 214).

Hay dos vías de relación claramente diferenciadas, aunque inevitablemente entramadas. No es posible la una sin la otra. Las empresas no pueden elegir recibir de su entorno las posibilidades $y$ limitaciones que este le ofrece ni tampoco no transmitir a este los efectos de su actividad. Hay una incidencia mutua, una relación dialéctica en definitiva. Aun así, hay en esto una constatación que, expresada de esta manera, resulta todavía muy abstracta. Es decir, hay un contexto espacial que induce efectos que van en doble vía pero que no precisa aspectos más concretos sobre cómo se realizan estos efectos, a quiénes afecta y, lo más importante quizás, cuáles son estos efectos. Por ahora, la afirmación de González hace posible constatar una condición esencial: la empresa habita un sistema abierto en el que interactúa con otros actores que están interconectados a través de marcos comunes y relaciones de interdependencia.

La empresa depende de su entorno, toma de él mucho de lo que requiere para realizar su actividad productiva. Al mismo tiempo lo transforma. No es viable, al menos no razonablemente, posicionarse desde una perspectiva que observe parcialmente esta cualidad sistémica de la existencia de la empresa. Aún más, tal 
como lo vemos, la empresa es en sí misma un sistema en el tanto que procesa insumos y genera productos, y que a su vez ocupa un lugar en un sistema más amplio. En toda esta compleja red, las relaciones son las que crean las conexiones. Pero, tal como se sugirió antes, estas conexiones son tanto elegidas como no-elegidas, así como sus efectos. Si, por ejemplo, solo se considera de una empresa A su impacto en el empleo al indicar el número de puestos de trabajo que genera de manera directa e indirecta, esto es insuficiente para valorar el conjunto de la incidencia de esa empresa en el espacio donde primariamente desarrolla su actividad.

Es la empresa un sistema, y es uno abierto en el tanto se alimenta a través de las interacciones e interrelaciones que genera para poder llevar a cabo su actividad productiva. Si se desea conocer más cercanamente los efectos de la actividad de una organización empresarial habría que iniciar teniendo claridad de esta cualidad sistémica. Para ello se hace necesario trazar una delimitación lo más precisa posible de los stakeholders o de las partes interesadas, que son quienes de manera más directa reciben los efectos de dicha actividad. El mapa puede ser tan complejo y amplio como cada empresa lo requiera, abriendo la posibilidad de crear grupos de actores, de tipologías de relación y, finalmente, de efectos. Para lograr esto último es necesario también determinar el para qué de esas relaciones, a qué necesidad o intención responden. En cualquier caso, los límites están determinados por la creatividad de quién investiga, es decir, de quién en última instancia desea conocer.

Hasta este punto se ha hecho referencia a cualidades o aspectos que comparten las empresas en general de cualquier tipo. Creemos que son cualidades que también comparten las organizaciones empresariales de la Economía Social y Solidaria. Esto, aunque cierto, no podría simplemente limitarse a una argumentación tan general de lo que son y cómo pueden relacionarse y afectar su entorno más próximo una empresa de tipo accionario a una de tipo asociativo-democrático. Hay diferencias constitutivas, que no son solo internas o que, al menos, no se expresan solo internamente. Sobre esto se hará referencia a continuación.

\section{EN QUÉ SENTIDO EL FENÓMENO ASOCIATIVO ES PARTICULAR}

Al decir que es un fenómeno particular, primeramente, se dice que lo es en símismo. Es decir, las organizaciones de Economía Social y Solidaria-también las cooperativas, desde luego- reúnen cualidades propias que las hace ser y pertenecer simultáneamente; o sea, ser identificables por esas cualidades comunes, particulares y compartidas. Hay tres elementos que podrían considerarse esenciales: propiedad colectiva, distribución democrática de los beneficios y gestión democrática. Luego, como es natural, cada uno de estos elementos se realiza con diversos matices. Esas cualidades que hacen que las organizaciones de la ESS se identifiquen dentro un "sector" común son, a la vez, las que las diferencian de otras formas de organización económica. Podría decirse que, sus cualidades propias adquieren una dimensión histórica relevante.

No se trata de entrar en argumentaciones valorativas, sobre si las organizaciones de la ESS son mejores a otras formas de organización empresarial. Por el momento se desea, únicamente, señalar esas características que las hace ser propiamente y ser diferentes en consecuencia. Estas diferencias tienen raíces que también son necesarias de dilucidar, aunque sea de manera muy breve. Esa raíz se refiere a las personas que emprenden -quiénes crean- y a relación de las personas con el tipo de proyecto productivo en cuestión -cómo se relacionan-, es decir, ¿quiénes son y cómo participan las personas de ese proyecto? Advertía Luis Razeto (2007) que detrás de los factores productivos hay personas concretas, así también el carácter de la empresa queda determinado dependiendo de cuál factor la organice. Por ejemplo, si es el capital, o sea, las personas que lo poseen, el factor organizador que tendrá una empresa de capital determinará los tres aspectos antes mencionados -propiedad, distribución de beneficios y tomas de decisiones- y quedarán irremediablemente determinados por este origen. De igual manera, las relaciones entre los factores y las personas que los poseen quedan condicionados.

En cambio, Razeto da un aporte para comprender las organizaciones de lo que denominó inicialmente "Economía de Solidaridad". Cuando las personas se unen para organizarse a pesar de poseer pocos y/o precarios factores de producción para desarrollar un proyecto productivo, ¿cómo se explica 
esto en términos económicos? ¿cómo se pasa de una precariedad de factores productivos a un proyecto viable? Para Razeto esto se explica por una categoría que llamó Factor $C^{5}$ (2007), que es la solidaridad, la cooperación, la ayuda mutua y la confianza convertidas en un concepto síntesis económicamente identificable. Esta funciona como categoría analítica y, en la práctica, como un factor productivo efectivamente operante en la empresa. Pero más allá de ellas mismas, habría que referirse a aquello que podría hacer que las organizaciones o empresas de Economía Solidaria incidan en espacio local.

\section{LA CUESTIÓN DEL ESPACIO Y EL ARRAIGO: CONDICIONES PARA UNA DINÁMICA RELACIONAL}

Las organizaciones de Economía Solidaria poseen algunas características que, como se verá, se consideran determinantes para sugerir un tipo de relación peculiar con el espacio local o zonas de influencia primaria ${ }^{6}$ (Céspedes, et al., 2017). Esto puede integrar varias comunidades y distritos, especialmente, aunque también cantones ocasionalmente. La cuestión que parece altamente relevante es determinar por qué se propone que estas organizaciones tienen una forma particular de relacionarse y, por tanto, de afectar positivamente sus entornos comunitarios.

Lo primero es considerar el espacio como una delimitación física, asociada indisolublemente a dinámicas particulares de tipo cultural, social, político y económico, en el cual, desde luego, existen las organizaciones de ESS. Así define y expresa Milton Santos lo que es el espacio: "conjunto indisoluble, solidario y también contradictorio, de sistemas de objetos y sistemas de acciones, no considerados aisladamente, sino como el contexto único en el que se realiza la historia" (Santos, 2000, p. 54). De este modo se entiende que el espacio es una unidad, que podría decirse compleja en el tanto integra objetos y acciones -entendiendo que las acciones son realizadas por sujetos, habría que incluirlos en el entramado-, que a la vez configuran y son configurados. Que una empresa, de cualquier tipo, se asienta en un espacio físico determinado, cae por su propio peso, es una obviedad. Pero, al comprender que una empresa o, como se refiere en este documento, una cooperativa o cualquier organización de la ESS, existe en un espacio particular, no se entiende como un hecho producto del azar o de la simple conveniencia por la ubicación de unos recursos. Las organizaciones de la Economía Social y Solidaria no se "instalan en" sino que "surgen desde" un espacio determinado. Esto es fundamental y es, tal como se propone, una cualidad particularmente potente en términos de explicar la diferencia entre nuestro objeto de estudio y otras formas de emprendimiento económico. Esta cualidad está asociada al segundo elemento que se considera determinante para comprender lo que hay de relevante en cómo se relacionan las organizaciones de ESS y las comunidades, el cual se aborda a continuación.

Este segundo elemento es el arraigo. Se trata de uno que, utilizado como categoría, cumple una función operativa fundamental en la tarea de enmarcar la peculiar relación de las organizaciones de ESS y el espacio local. Por arraigo entendemos aquella cualidad entre dos elementos, $A$ y $B$, en la que $B$ no puede ser entendida $\sin A$. Es decir, A (espacio local) existe y a la vez define la posibilidad de existencia de B (actor social en cuestión). Hay un vínculo de dependencia que se teje a todo nivel: social, político, cultural y económico, de $B$ con $A$. En definitiva, $B$ no puede ser entendida $\sin A$. No se trata de un vínculo fortuito, ni si quiera voluntario. Es así como B se enraíza en A para poder ser y cumplir su propósito específicamente económico que es el que le da, finalmente, su razón de ser, pero entendiendo que, en el proceso de lograrlo, este está configurado -y configurando- por los condicionamientos y posibilidades de todo tipo que $\mathrm{A}$

5. El Factor C merece sin duda un análisis aparte, amplio y detallado. Se puede encontrar a lo largo de la obra de Razeto, un texto en particular es "Lecciones de Economía Solidaria. Realidad, teoría y proyecto" (Uvirtual, 2007).

6. Se refiere al espacio inmediato más cercano a la organización, definido por estos autores a partir de la procedencia de las personas asociadas. 
le provee. Decimos entonces que las organizaciones de ESS se arraigan en un espacio determinado, porque lo necesitan sin remedio y esto está determinado porque ellas, como se decía más arriba, surge desde él. En este sentido el espacio es un ente vivo que da a luz a la organización, y esta porta la información genética que este le ha impreso.

La investigación "Cooperativas y su impacto al desarrollo. Reconociendo formas y vías de impacto" (Céspedes et al., 2017), detecta lo que los autores llaman roles sociales de las cooperativas. Es decir, al buscar de qué forma las cooperativas ${ }^{7}$ contribuyen al desarrollo en las llamadas zonas de influencia primaria, los investigadores encuentran que no se trata solo de "impactos", entendido como incidencias puntuales, sino de una relación estable y sostenida que podría entenderse como un resultado del arraigo de las cooperativas al espacio que las ha generado y en cual existen. Es decir, hay una pertenencia o identificación profunda que permite que se generen vínculos en la forma de roles que estas ejercen y que crean una dinámica de relación que posibilita diversos aportes al desarrollo de las comunidades. Esto es posible sostenerlo, en el tanto el trabajo antes mencionado, identifica roles que permiten comprender que esos aportes de las cooperativas obedecen tanto a una cierta estabilidad como a un re-conocimiento mutuo -cooperativa / comunidad. Sobre esto vale la pena profundizar un poco más.

Los hallazgos y el sustento que provee este trabajo permiten, en este punto concreto, comprender que roles como lo son, por ejemplo, "agente detector de necesidades locales" y "plataforma generadora de insumos (información) a terceros sobre problemáticas locales", suponen más que una relación puntual, refieren a una conexión entre las cooperativas y las comunidades. Ahora bien, ¿cómo detectan y llegan a estos hallazgos los investigadores? Hay en esto dos elementos que ayudan a comprenderlo: el primero se refiere a que estas organizaciones se identifican con las comunidades y esto les permite no ser indiferentes a sus problemáticas. Las conocen. El segundo elemento refiere a comunicar ese conocimiento. Esto es especialmente relevante en el tanto plantea una pregunta que es clave: ¿cómo y con qué autoridad las cooperativas comunican esta información? Bueno, esto refiere a la legitimidad que tienen estas organizaciones frente a terceros que confían en la información que ellas ofrecen sobre la comunidad. Es decir, terceros, que pueden ser públicos o privados, reconocen el arraigo de las cooperativas, el cual se traduce en la capacidad de percibir y reconocer problemáticas locales para luego comunicarlas. También es posible plantear la situación de manera inversa, ¿podría un actor externo solicitar información a una organización que no evidencia conocimiento de las situaciones que vive la o las comunidades? En esta confianza hay, implícitamente, un reconocimiento del arraigo y de la capacidad de conocer el entorno comunitario que poseen las cooperativas ${ }^{8}$.

Estas cualidades no surgen espontáneamente ni en cualquier condición. Quienes integran las organizaciones de la ESS son, simultáneamente, quienes habitan estos espacios. Esto es así comúnmente. Podría decirse que una fuerte característica que marca el carácter local de estas organizaciones está dada por esta doble pertenencia. Citando nuevamente el trabajo de Céspedes y Segura, se encontró que era frecuente en los casos estudiados, que refirieran a solicitudes de colaboración que las mismas comunidades solicitaban para apoyar actividades comunitarias, como lo indica el rol "Plataforma de apoyo a actividades que recaudan fondos en beneficio de las comunidades o la región". Es decir, no solo actores externos reconocen esa conexión entre las cooperativas y las comunidades, también estas últimas lo hacen. Esto explica la legitimidad local y hacia afuera que tienen estas organizaciones. Este estudio permite visibilizar cómo se materializan las relaciones entre las cooperativas y las comunidades, pero además permite comprenderlas como una dinámica estable en el tiempo que además supone vínculos entre ellas que no son habitualmente visibles ni visibilizados. El arraigo al espacio local podría decirse que genera pertenencia por una parte y reconocimiento por otra. Ambas cualidades caracterizan una forma de relacionarse y consecuentemente de incidencia que podría considerarse peculiar, diferente a otras formas de relación que otros actores, incluso otros tipos de organización económica pudieran tener.

7. Este estudio se realizó considerando solo cooperativas; once en total. Aunque la población a la que se hace referencia en este documento incluye a las cooperativas, no solo se refiere a estas. Dentro de las organizaciones de la Economía Solidaria se incluyen diversidad de formas asociativas de carácter democrático.

8. Cuando se hace referencia a esta investigación, se habla de cooperativas explícitamente porque los casos de estudio correspondieron a estas organizaciones en particular. Aun así, podemos sostener que las cualidades y la composición de cualquier organización de carácter permite extender estas observaciones al fenómeno asociativo, sino de manera idéntica, sí aproximada. 
VII. HACER VISIBLE LA

PARTICULAR RELACIÓN DE LAS ORGANIZACIONES DE LA ESS CON LAS COMUNIDADES

\section{La dinámica relacional como puerta de entrada}

Se ha insistido en mostrar que las empresas, y también las organizaciones de la ESS, conviven como parte de un sistema abierto más amplio, que las condiciona de muchas maneras, al tiempo que se produce un efecto producto de la actividad de estas que recibe de vuelta ese sistema más grande. En síntesis, una relación dialéctica. Esta cualidad dual es en realidad generalizable a cualquier empresa, pero, también se ha señalado, que elementos internos de las organizaciones de ESS no solo las caracteriza, sino que también les permite crear vías de relación particulares con sus entornos. Estas formas particulares de relación propias de estas organizaciones son las que es necesario conocer, precisamente porque ellas conducen a conocer los efectos que estas generan en sus zonas de influencia primarias, o sea, las comunidades.

La propuesta que se ha desarrollada tiene como corolario esta tesis: si se desea conocer las formas de relación y los efectos de las organizaciones de la ESS, especialmente los efectos extra-económicos de estas, se debe responder inicialmente a una pregunta que es central y que se presenta como la síntesis de lo que se ha venido desarrollando: ¿con quiénes se relacionan las organizaciones de la ESS y para qué? El supuesto ${ }^{9}$ del que se parte es que, al conocer con quiénes se relacionan las organizaciones, es posible determinar cuáles son esas vías de incidencia y qué propósito tienen, de este modo se construye una vía de aproximación metodológica a la cuestión de conocer el efecto de las organizaciones de Economía Social y Solidaria. Con mucha facilidad plantearse este objetivo puede resultar confuso de inicio, especialmente si se trata de conocer los efectos más allá de lo entendido como "económico". ¿Cómo y por cuáles medios se pueden identificar? Es la pregunta que pareciera marca un punto de partida y a la cual se ha quiere dar una salida desde esta propuesta. Es así como, fijar la atención en la dinámica relacional viene a ser una puerta de entrada, metodológicamente hablando, que permite satisfacer el objetivo antes mencionado.

\section{LA DINÁMICA RELACIONAL Y LOS EFECTOS SOCIALES (EXTRA- ECONÓMICOS) DE LAS ORGANIZACIONES DE LA ESS: DOS CASOS DE APLICACIÓN}

Los efectos sociales de las cooperativas $y$, en general, de las organizaciones de la ESS son múltiples y superan lo estrictamente económico. Se han ofrecido criterios teóricos para sostener el sentido que tiene comprender a las organizaciones como sistema abiertos que, a su vez, se desarrollan como parte de un sistema más amplio. Esto permite ver la riqueza y la complejidad de relaciones posibles. Pero, aun así, no se trata de un caos de relaciones que van y vienen sin sentido. Cuando se comprende que el factor espacial es determinante, tanto para promover el surgimiento y desarrollo de organizaciones productivas como para comprender las posibilidades de incidencia local que estas tienen, se abren posibilidades para abrir nuevas dimensiones de comprensión más amplias de lo estrictamente entendido como económico. Las relaciones económicas son antes relaciones sociales, pero, además, no se dan en el vacío. Buscar, así, sin más, los efectos sociales de las organizaciones de la ESS, presenta una primera línea de dificultad que parece ser metodológica, pero creemos que es primeramente teórica. En todo caso, este recorrido que se ha procurado hacer, es oportuno llevarlo a un nivel de comprobación empírica, aunque sea de manera preliminar.

El instrumento utilizado intenta conocer de manera general dos aspectos, en este orden: con quiénes y para qué se relacionan las organizaciones. Esto se combina con preguntas específicas, para conocer en qué consisten estas interacciones, a quiénes se dirigen, a qué necesidad responden, principalmente. También es importante conocer si las acciones en cuestión se originan de una petición externa o una iniciativa interna, esto para identificar de alguna manera lo que podría entenderse como "legitimidad" comunitaria de la organización, pues las solicitudes externas a las organizaciones se entenderían como una forma en que la comunidad reconoce la existencia y el vínculo con la organización. De igual forma interesa conocer la frecuencia para determinar si se trata de una acción puntual o más bien una condición estable que lleva a la organización a fungir una especie de rol comunitario.

9. No se trata de un supuesto arbitrario, surge como producto de las dinámicas de relación detectadas en el estudio antes citado (Céspedes y Segura, 2017). 
Además de lo anterior, también es útil conocer si la acción tiene un seguimiento y alguna forma de contabilización por parte de la organización. Se ha visto que con frecuencia las organizaciones no contabilizan ni dan seguimiento, lo que genera que estas relaciones y sus efectos se desconozcan por parte de ellas mismas. Esto dificulta tener datos sobre el aporte a la comunidad que las organizaciones de carácter asociativo realizan, por ello resulta propicio, partir de conocer los medios, con quién y para qué se relaciona la organización. Hay que procurar hacer emerger a la superficie dinámicas -y sus efectos- habitualmente invisibilizadas. Es este un principio metodológico que podría ayudar a sacar de la sombra los aportes extra-económicos, que a su vez permitiría conocer mejor el rol de las organizaciones asociativas con arraigo comunitario; desde luego, no solo de aquellas bajo la figura de cooperativas.

El primer caso es el de Coopepan ${ }^{10}$, una cooperativa de autogestión con más de 30 años de existencia, nace exactamente en setiembre de 1981 y surge a partir del cierre de la Panificadora San Carlos ubicada en Ciudad Quesada, San Carlos. Los empleados toman la empresa y la transforman en una cooperativa, cosa que fue posible mediante el apoyo financiero del Infocoop y de la Unión de Cooperativas de la Zona Norte (URCOZÓN). Hoy en día, después de varias etapas, incluso de expansión, Coopepan cuenta con un solo local en Ciudad Quesada de San Carlos y está integrada por 12 personas asociadas. Por tratarse de una cooperativa autogestionaria, las personas asociadas son simultáneamente trabajadoras.

En tanto, el segundo caso, se trata de Coopeande $\mathrm{N}^{\circ} 7$ (Coopeande), una cooperativa fundada el 3 de julio de 1967 por cincuenta maestros con un capital social de $\$ 3500.00$ (tres mil quinientos colones) bajo el giro de ahorro y crédito. En la actualidad es una cooperativa de servicios múltiples y su principal actividad sigue siendo el crédito. Su cartera de créditos es de \$4.024 millones, cuenta con 22 funcionarios laborando tiempo completo y la Asamblea se realiza por delegados en el mes de abril. En el 2017 la cooperativa celebra sus cincuenta años con más de mil asociados que residen en más de 23 localidades del país. Su sede central se encuentra ubicada en Aguas Zarcas, distrito del cantón de San Carlos, Zona Norte del país.

Uno de los elementos importantes que se ha querido tener en cuenta al momento de elegir estos casos, es que las empresas cooperativas tuvieran una comprobada estabilidad. Como se puede ver, ambos casos guardan una distancia importante en cuanto al tipo de actividad y al tamaño. Esta diversidad es la constante al observar el conjunto del sector cooperativo nacional que, por ejemplo, el Censo Cooperativo de 2012 (Estado de la Nación / INFOCOOP) distribuía en 17 grandes sectores productivos. A este criterio hay que agregar el de "modelo de cooperativa", a saber: tradicional, autogestionaria, integración y cogestionaria. Los casos elegidos se encuentran en el tipo autogestionaria (Coopepan) y tradicional (Coopeande $\mathrm{N}^{\circ}$ 7). La cuestión que debiera quedar clara es que, aun agrupando a las cooperativas por estos grandes criterios, habría diferencias entre aquellas que se encuentren compartiendo uno o más criterios de agrupación. Tanto Coopepan como Coopeande son distintas tanto en lo que respecta al modelo como al área productiva en el que se desarrollan. A pesar de ello, como se verá, sus cualidades de arraigo territorial y relación con el espacio concreto donde desarrollan centralmente su actividad, comparten elementos que son los que interesan y sobre los cuales se pondrá especial atención.

\section{Profundizar en los casos}

Inicialmente habría que aclarar que no corresponde seguir una lógica comparativa entre los casos. De partida se entiende que son casos distintos en sus características, nos interesa observar similitudes o diferencias en los modos y vías de relación con el entorno, no entre las cooperativas observadas. Evidentemente hay aspectos que valdrá la pena preguntarse por qué se dan una manera u otra según cada caso, pero, repetimos, no con el fin de asociar esto con una valía entre los casos que no viene al caso establecer. Por otro lado, se ve que el carácter que imprime la "esencia cooperativa" es posible detectarlo también en la naturaleza comunitaria que evidencian estas formas y vías de relación que hemos destacado para ambos casos. Es una casualidad que tanto Coopepan como Coopeande hayan registrado un total de 12 "vías o formas de incidencia". Las consultas se hicieron con varios meses de distancia, aunque el instrumento utilizado fue el mismo. De todas formas, esto no explicaría el porqué de esta coincidencia. Se trata, simplemente, de una casualidad.

\section{Agrupar por áreas de incidencia}

Resulta útil ubicar con algún criterio las "formas y vías de relación" que generan impactos específicos, en este caso se hizo según las dimensiones del desarrollo (PNUD, 1990), a saber: salud, educación o ingreso y se agregó una cuarta: "organización y bienes comunitarios". Esta lógica de tipificación de las formas y vías de relación permiten asociar el actuar de las cooperativas con incidencias concretas en

10. Los datos de los casos se recogieron mediante la aplicación de dos entrevistas, realizada a la señora María Elena Rodríguez, actual gerente de esta cooperativa. La entrevista se realizó el viernes 20 de enero de 2017 en las instalaciones del Instituto Nacional de Fomento Cooperativo en Barrio México, San José. 
dimensiones del desarrollo, con las más clásicas, desde que el PNUD las introdujo, al menos. Nos parece que son lo suficientemente amplias y a la vez ordenadas para agrupar con un criterio adecuado el conjunto de hallazgos. Desde luego, otros criterios pueden ser utilizados, según interés u otras perspectivas teóricas. Al mismo tiempo, tomamos de Céspedes y Segura (2017) la dimensión ad-hoc que utilizaron en su trabajo, y que llamaron: bienes y servicios públicos / capacidades colectivas. Nos parece que resumir esta dimensión como organización y bienes comunitarios simplifica por una parte y, por otra, conserva el espíritu de utilidad que quisieron recoger los autores con esta creación analítica que tiene su inspiración en aportes de Amartya Sen (2000). Este autor reconoce el aporte de los servicios públicos al desarrollo humano en el tanto permiten un disfrute democrático de la población de un bien o servicio al cual individualmente no se podría tener acceso. Se reconoce, de esta manera, el valor que tiene lo público que, en la propuesta de Céspedes y Segura, se amplía a lo colectivo, para evitar asociaciones restringidas de lo público a lo estatal. Con esto se entiende que hay elementos comunitarios noestatales.

Tabla 1. Vías /formas de relación por cooperativa según cada una de las dimensiones deldesarrollo de propuestas.

\begin{tabular}{|c|c|c|c|}
\hline & Coopepan & Coopeande & Total \\
\hline Salud & 1 & 2 & 3 \\
\hline Educación & 4 & 3 & 7 \\
\hline Ingreso & 1 & 1 & 2 \\
\hline $\begin{array}{c}\text { Organuzación } \\
\text { y bienes } \\
\text { comunitarios }\end{array}$ & 6 & 4 & 10 \\
\hline Género ${ }^{11}$ & 0 & 2 & 2 \\
\hline Total & 12 & 12 & 24 \\
\hline
\end{tabular}

Fuente: Elaboración propia sobre la base de datos de la ONEl, 2017.

Lo primero que se quisiera señalar es la coincidencia con los casos estudiados por Céspedes y Segura en el trabajo ya mencionado. Hay una notable predominancia de la dimensión asociada a lo colectivo. En dicho estudio ya se evidenciaba que esta dimensión era la más impactada de manera primaria por la acción externa (relaciones) de las cooperativas.
En esta pequeñísima muestra esto se confirma de manera categórica. Pareciera haber una especie de "conexión natural" de las cooperativas estudiadas con todo aquello que se considera de provecho común. Sin duda esto parece relevante.

Hay que recordar que las cooperativas inciden en el ingreso en el tanto generan empleo o excedentes (si es que los hay). Pero acá, lo observado, es únicamente aquello que es producto de la relación de la cooperativa como agente local en su relación con ese universo más amplio que es la comunidad. Lo que sucede al interior de la cooperativa, aunque relevante, no es parte de lo que este abordaje permite reconocer. Precisamente, el objetivo de hacerlo de este modo, se debe a la necesidad de dar cuenta de aquellos aspectos más habitualmente invisibilizados o poco comprendidos de cómo y en qué medida las cooperativas inciden en las comunidades. Como se ve, y confirma los resultados de otros 11 casos estudiados por Céspedes y Segura (2017), las dimensiones salud y educación son las menos impactadas $y$, de ellas, la menos es salud. No es un rol que propiamente ejerzan las cooperativas, especialmente en un contexto como el costarricense en el que el Estado tiene una presencia relevante en esta materia; en el caso de la educación el nivel de incidencia podría catalogarse como medio.

El tercer aspecto que se desea mencionar es la inclusión obligada de la dimensión "género". Esta dimensión, la cual nos parece que no admite discusión en un enfoque inclusivo, tal como se puede comprender hoy en día, se incluye a partir del hallazgo evidenciado empíricamente. Es Coopeande la que de manera consciente apoya acciones dirigidas a la promoción de las mujeres. Por su especificidad, no es posible considerar esta dimensión dentro de alguna de las otras 4 propuestas inicialmente. Por ejemplo, la dimensión ambiental bien puede ser incluida dentro de "bienes comunitarios", porque de hecho la naturaleza admite en alguna medida esta comprensión. Vale recordar la categoría ampliamente estudiada por Eleonor Ostrom (1990) a lo largo de su carrera y popularizada con su libro "Los bienes comunes". Estos hacían referencia a la naturaleza en el tanto era un recurso susceptible de uso y apropiación colectiva. Al menos podría caber una doble categorización sobre esto los "bienes naturales" de los que disfrutamos colectivamente, en tanto, la categoría género no admite esta doble posibilidad de categorización.

\section{La legitimidad como un hallazgo trascendente}

Resulta necesario indicar cómo ser observó

11. Coopeande, en dos de las vías o formas de incidencia detectadas, mostró expresamente su vinculación con actividades relacionadas a la promoción de las mujeres. Por esta razón se ha tenido que agregar la dimensión "género", porque evidencia preocupaciones con un tema que no estaba contemplado pero que, cae por su propio peso la relevancia que tiene cuando se habla de desarrollo humano. Se trata de una reivindicación en auge y que ha servido para evidenciar la deuda histórica, a todo nivel, con las mujeres. 
empíricamente "la legitimidad" y cómo se entiende en este contexto. Primero vale aclarar que la legitimidad se entiende como la validación que terceros, sobre todo locales, hacen de la cooperativa. Esta validación que acaba legitimando a las cooperativas como un actor comprometido con el desarrollo de las comunidades, se evidencia cada vez que comprobamos que es la cooperativa la que es interpelada desde afuera para que se comprometa o colabore de cualquier manera con las comunidades. Hay en esto un reconocimiento no solo de la existencia de las cooperativas sino también de unos valores que hace que estas se comprometan en un alto grado con las necesidades y requerimientos diversos de las comunidades. A continuación, veremos el origen (quién solicita) las formas de interacción que acabamos identificando y consignando como vías y formas de incidencia.

Tabla 2. Vías y formas de incidencia, según quién las solicita.

\begin{tabular}{|c|c|c|c|c|}
\hline & $\begin{array}{c}\text { La propia } \\
\text { cooperativa }\end{array}$ & $\begin{array}{c}\text { Un } \\
\text { extremo }\end{array}$ & Mixto & Total \\
\hline Coopepan & 1 & 11 & - & 12 \\
\hline Coopeande & 2 & 6 & 4 & 12 \\
\hline Total & 3 & 17 & 4 & 24 \\
\hline
\end{tabular}

Fuente: Elaboración propia a partir de información primaria.

El dato más débil, en términos numéricos, indica que las cooperativas en realidad solo 3 veces de 24 , o sea, $12,5 \%$, tiene la iniciativa de la iniciar la vía de incidencia. Por otro lado, el 70\% de las veces es un actor externo el que propicia la vía de incidencia. Es en este dato en el que nos basamos para sostener que las cooperativas estudiadas son agentes altamente legitimados a nivel local. Esto había sido un hallazgo surgido del proceso de entrevistas realizado en el estudio antes mencionado, que ahora se confirma. Ahora, en este breve ejercicio, la pregunta se incluyó de manera explícita y la evidencia se consolida, incluso de manera abrumadora.

La siguiente casilla: "mixto", refiere a que en ocasiones es un agente externo quien solicita o bien, un delegado de la Cooperativa que participa en una instancia colegiada afín al tema o a la institución que solicita. En estos casos, quienes fungen como representantes de la Cooperativa en ese espacio sirven de "puente". Esta particularidad es altamente interesante, sobre todo si se plantea la pregunta: ¿Por qué las cooperativas tienen representación en instancias externas de tipo social, cultural, económico o político? Al parecer formar parte de estas instancias representa en sí mismo otro modo de legitimación de las cooperativas y de su rol en las comunidades. Hay implícito un reconocimiento de las cooperativas como un actor relevante.

\section{Los efectos sociales de las organizaciones de la ES}

La evidencia recabada en estos dos casos, no es porsímisma concluyentecomo prueba de una tendencia que sea generalizable para todas las organizaciones de la ESS. Pero, si se toman estos resultados y se contrastan con los obtenidos de la investigación más amplia (11 casos) que se ha venido citando, se confirma que en primer lugar lo relacionado con lo público / colectivo es la dimensión que más frecuentemente suelen afectar las cooperativas estudiadas. En segundo lugar, se encuentra la dimensión educación. Es decir, tenemos un patrón que se repite en dos nuevos casos. Esto sin duda es una cuestión relevante al menos en dos sentidos. El primero, porque permite reconocer la cuestión de por qué organizaciones con un carácter colectivodemocrático tienden a afectar con mayor peso aquellos aspectos asociados a los bienes, servicios y/o saberes comunes. Sería como una especie de continuo o efecto continuado derivado de la naturaleza interna o el carácter propio constitutivo de las cooperativas y de las organizaciones de la ESS. Lo segundo, que permite abrir el espacio para preguntarse cuáles son los motores que inducen este comportamiento, esta forma de incidencia. No son cuestiones que propiamente se puedan responder en el contexto de este artículo, pero son cuestiones sugerentes que emergen a la luz de la evidencia que va surgiendo con estos ejercicios de aproximación empírica.

\section{A MANERA DE CONCLUSIÓN}

Comprender la incidencia de los actores sociales, cualesquiera que sean, supone comprenderlos en sus contextos y dinámicas. Por ello hemos querido proponer una vía de comprensión teórica de las organizaciones económicas en general y, en particular, de aquellas de carácter asociativo-democrático. Se ha querido explicitar una perspectiva teórica que, aunque toma su punto de partida de la teoría de los 
stakeholders, intenta ir más allá al incorporar otros elementos que abren un margen de comprensión más amplia del fenómeno empresarial, hasta llevarlo al mundo de las organizaciones de la ESS en concreto. No solo se trata de una diferencia instrumental, limitada a un modo de gestión, de distribución de beneficios o de propiedad, sino directamente vinculada con la relación que las personas emprendedoras tienen con el espacio desde el cual surge el proyecto productivo de carácter asociativo. Una vez que se esclarece que ellas poseen esta doble pertenencia, a la organización y a la comunidad, se abre una puerta de comprensión ampliada del fenómeno económico asociativo. No es lo mismo instalarse en un territorio que emprender desde él por una pertenencia vital e histórica. Esta doble pertenencia de quienes conforman un proyecto productivo de estas características es un elemento explicativo muy potente en el sentido de que ofrece una vía de comprensión ampliada y más cercana a la realidad de las organizaciones de la ESS.

Tal como propone la teoría de los stakeholders, existe para cualquier empresa "partes interesadas" más allá de los directamente vinculados a la empresa: accionistas y trabajadores. La concepción de las empresas como sistemas abiertos lleva, invariablemente, a este dibujo ampliado del rango de influencia social de una organización empresarial, sea del tipo que sea. Aún así, aunque aporta un valioso principio teórico, no acaba de explicitar por sí misma el modo en que las partes se relacionan. En efecto, las partes se relacionan por medio de trazos que se materializan en forma de canales de convergencia que deben ser estudiados, comprendidos y explicados. Por ello es tan relevante agregar a este aporte teórico una perspectiva que señale sin ambages esas vías de conexión, lo que en este artículo se ha querido llamar enfoque relacional. Cada teoría se plantea sus propios objetivos y parte de otras explicaciones parciales, ante las cuales responde para dar un paso al frente. En este sentido, la teoría que se ha tomado como punto de partida es sin duda un avance importante que se nutre aún más cuando se puede complementar con una perspectiva que logre no solo reconocer que hay otros actores involucrados en la actividad de las empresas en tanto actores sociales, sino que estos vínculos ocurren materialmente por medio de las relaciones. Es decir, no se trata de partes interconectadas fortuitamente o de manera esporádica. Resulta altamente relevante conocer estas conexiones que a su vez abren posibles nuevas explicaciones del espacio social más amplio que integran las empresas y las organizaciones de la ESS. En efecto, las partes se relacionan, se afectan mutuamente en todas las direcciones y con muchos actores involucrados, esto propiamente debe ser explicitado y estudiado, también para conocer los efectos de un actor en particular, pero no solo. Las explicaciones derivadas pueden ampliarse grandemente y permitir un mejor conocimiento del espacio social del que las organizaciones forman parte.

Para la ESS y para el cooperativismo como el sector de mayor trazado histórico en Costa Rica, es importante valorarse como sector social y económico más allá de los aportes puntuales de una o de otra organización, por importante que sea. El aporte visibilizado como conjunto puede abrir paso a posicionamientos políticos e ideológicos de mayor peso ante otros actores relevantes del contexto nacional e internacional. Ahora bien, poder apreciar el conjunto no necesariamente supone un salto a datos agregados o sumamente abstractos. Alcanzar niveles de desarrollo teórico más evolucionados en esta línea, podría dar lugar a herramientas de análisis territorial del sector que profundice en las vías de relación no solo entre organizaciones de la ESS sino, en general, con otros actores locales, incluyendo desde luego a las instituciones del Estado. De esta manera se podría pensar en informes desagregados territorialmente que tendrían el potencial de convertirse en insumos relevantes tanto para la creación de política intrasectorial como de aquella que las diferentes instituciones del Estado pueden diseñar y dirigir para el promoción del sector $y$, sobre todo, del impacto al desarrollo local que generan las organizaciones económicas de carácter asociativo-democrático con arraigo comunitario y regional. 


\section{BIIBLIOGRAFÍA}

Bobbio, N. (2001). El futuro de la democracia. México. Fondo de Cultura Económica.

Céspedes, J. C. y Segura, Ó. (2017). Impacto de las Cooperativas al Desarrollo. Reconociendo formas y vías de impacto. San José. Editorial Infocoop.

Chaves, J.A. (1999). De la Utopía a la política económica. Para una ética de las políticas económicas. Salamanca, España. Editorial San Esteban.

Cortina, A. (1997). Presupuestos éticos del quehacer empresarial. En: Adela Cortina (coord.), Rentabilidad de la ética para la empresa. España. Fundación Argentaria.

El Mundo (2013). La tragedia en Bangladesh destapa 'los talleres de la miseria.' [Citado el 4/8/2017]. Recuperado de http://www.elmundo.es/elmundo/2013/04/25/ internacional/1366885756.html

Friedman, M. (1966.). Capitalismo y libertad. Madrid. Ediciones RIALP.

González, E. (2007). La teoría de los stakeholders. Un puente para el desarrollo práctico de la ética empresarial y de la responsabilidad social corporativa. Veritas, vol. II, n 17, pp. 205-224. Recuperado de http:// www.redalyc.org/pdf/2911/291122924002.pdf

Programa Estado de la Nación en Desarrollo Humano Sostenible (2013). Decimonoveno Informe Estado de la Nación en Desarrollo Humano Sostenible. San José.

Razeto, L. (2007). Lecciones de Economía Solidaria: Realidad, Teoría y Proyecto. Santiago de Chile. Ediciones Uvirtual.net.

Santos, M. (2000). La Naturaleza del Espacio. Técnica y tiempo. Razón y emoción. Barcelona, España. Editorial Ariel S.A. 